Comment. Math. Helv. 73 (1998) 45-70

0010-2571/98/010045- $26 \$ 1.50+0.20 / 0$
(C) 1998 Birkhäuser Verlag, Basel

Commentarii Mathematici Helvetici

\title{
A vanishing theorem for modular symbols on locally symmetric spaces
}

\author{
Toshiyuki Kobayashi and Takayuki Oda
}

\begin{abstract}
A modular symbol is the fundamental class of a totally geodesic submanifold $\Gamma^{\prime} \backslash G^{\prime} / K^{\prime}$ embedded in a locally Riemannian symmetric space $\Gamma \backslash G / K$, which is defined by a subsymmetric space $G^{\prime} / K^{\prime} \hookrightarrow G / K$. In this paper, we consider the modular symbol defined by a semisimple symmetric pair $\left(G, G^{\prime}\right)$, and prove a vanishing theorem with respect to the $\pi$-component $(\pi \in \widehat{G})$ in the Matsushima-Murakami formula based on the discretely decomposable theorem of the restriction $\left.\pi\right|_{G^{\prime}}$. In particular, we determine explicitly the middle Hodge components of certain totally real modular symbols on the locally Hermitian symmetric spaces of type IV.
\end{abstract}

Mathematics Subject Classification (1991). Primary 11F67; Secondary 22E46, 53C55, $22 \mathrm{E} 40$.

Keywords. Modular symbols, semisimple Lie group, Zuckerman-Vogan module, MatsushimaMurakami formula, modular varieties, discrete decomposable restriction, bounded symmetric domain, discontinuous group, symmetric space.

\section{$\S 1$. Introduction}

Our concern in this paper is to describe the middle Hodge components of the Poincaré duals of certain $2 n$-cycles on the arithmetic quotients $V=\Gamma \backslash D$ of the bounded symmetric domain of type IV

$$
D \cong S O_{0}(2 n, 2) /(S O(2 n) \times S O(2))
$$

where the $2 n$-cycles are defined via the embedding $S O_{0}(2 n, 1) \hookrightarrow S O_{0}(2 n, 2)$. These cycles are special cases of generalized modular symbols. To explain our motivation of this investigation, let us recall the origin of modular symbols briefly.

Historically speaking, the idea of modular symbols was introduced and extensively studied for elliptic modular curves $C=\Gamma \backslash \mathfrak{H}(\mathfrak{H}$ is the complex upper half plane) in the work of Manin-Drinfeld and Mazur-Swinnerton-Dyer. Modular symbols are defined to be chains or cycles on the compactification $C^{*}$ of $C$, obtained as the images of arcs on $\mathfrak{H}$ joining two cusps with respect to $\Gamma \subset S L_{2}(\mathbb{Z})$. 
In terms of them, the first homology group $H_{1}\left(C^{*}, \mathbb{Z}\right)$ is quite explicitly computed. The theory is "elementary" and has applications to the special values of $L$-functions and to the construction of $p$-adic $L$-functions of modular forms. The same cycles appear very naturally in the theory of half-integral weight modular forms (cf. Shintani [21]).

There have been attempts to push forward the investigation since the late '70's for higher dimensional arithmetic quotients, i.e. to introduce the object called generalized modular symbols given as follows.

Let $G$ be a semisimple Lie group and $\Gamma$ a discrete subgroup such that the volume $\operatorname{vol}(\Gamma \backslash G)<\infty$. Let $G^{\prime}$ be a closed subgroup of $G$ such that $\Gamma^{\prime}:=G^{\prime} \cap \Gamma$ is also discrete and co-volume finite in $G^{\prime}$ (this paper will be restricted to the cocompact cases). Then, the inclusion map induces a map of real analytic varieties of double cosets:

$$
\iota: \Gamma^{\prime} \backslash G^{\prime} / K^{\prime} \rightarrow \Gamma \backslash G / K
$$

where $K$ is a maximal compact subgroup of $G$ and $K^{\prime}:=K \cap G^{\prime}$. The image of $\iota$ defines a chain $C_{\left(G^{\prime}, \iota\right)}$ on $\Gamma \backslash G / K$, and when $\Gamma \backslash G$ is compact, $C_{\left(G^{\prime}, \iota\right)}$ is a cycle on $\Gamma \backslash G / K$. When $\Gamma \backslash G$ is non-compact, $C_{\left(G^{\prime}, L\right)}$ is sometimes compactified to give a cycle on a natural compactification of $\Gamma \backslash G / K$.

There are a number of results on generalized modular symbols. In some special cases, the periods of automorphic forms along generalized modular symbols are represented as the special values of automorphic $L$-functions (cf. [4], [18]). Though it is desirable to generalize this type of results, our current knowledge on generalized modular symbols is still very poor. Presently, many of literatures on generalized modular symbols are concerned only in establishing the non-vanishing of these cycles, using the intersection numbers of these cycles (cf. [1]), or using the Weil representation (cf. [11], [13]).

Our main results and the method of the proof are quite different from these previous works. First, we shall show the vanishing of the "non-trivial part" of the $(n, n)$-components of certain modular symbols. Secondly, the method of our proof is based on a recent theory of unitary representations on the discrete branching laws by T. Kobayashi ([6], [7], [8], [9]). An explicit vanishing theorem for the modular symbols corresponding to $G^{\prime} \hookrightarrow G$ is then obtained from the criterion on the (non-)existence of continuous spectrum of an irreducible unitary representation of $G$ with non-zero continuous cohomologies when restricted to a non-compact reductive subgroup $G^{\prime}$. Then, this method leads us to some heuristic argument that the usual method of the Rankin-Selberg convolution for the construction of automorphic $L$-functions of non-trivial $(n, n)$-type harmonic (automorphic) forms $\omega$ on $V$ gives only zero, hence it gives no information for the periods of non-trivial (n, n)-type harmonic forms on $V$ (cf. Remark 6.1).

Our main results (see Theorem 2.8 and Theorem 4.3) will be formulated for a real reductive Lie group in general. But, we illustrate them by the most interesting case: Let $G / K=S O_{0}(2 n, 2) / S O(2 n) \times S O(2)$ be the Hermite symmetric space of type IV. Its dual symmetric space is $G_{U} / K:=S O(2 n+2) / S O(2 n) \times$ 
$S O(2)$, that is, the complex quadric. The $2 n$-th de Rham cohomology group $H_{\mathrm{de} \operatorname{Rham}}^{2 n}\left(G_{U} / K ; \mathbb{C}\right)$ is a two-dimensional vector space, with generators $[\xi]:=$ $\left[\wedge^{n} \kappa\right]$ and $[\eta]$. Here $\kappa$ is the Kähler form on $G_{U} / K$ and $\eta$ is a $G_{U}$-invariant primitive differential form. We note that both $[\xi]$ and $[\eta]$ lie in the $(n, n)$-type Hodge components of $H_{\mathrm{de} R h a m}^{2 n}\left(G_{U} / K ; \mathbb{C}\right)$ and that $(p, q)$-type Hodge components of $H_{\mathrm{de} \text { Rham }}^{2 n}\left(G_{U} / K ; \mathbb{C}\right)(p+q=2 n)$ are non-zero if and only if $p=q=n$.

Suppose $\Gamma$ is a discrete, torsion-free co-compact subgroup of $G$. Then there is a natural injective map of cohomology groups:

$$
\Phi: H_{\text {de Rham }}^{*}\left(G_{U} / K ; \mathbb{C}\right) \rightarrow H_{\text {de Rham }}^{*}(\Gamma \backslash G / K ; \mathbb{C}) .
$$

Elements in the image of $\Phi$ are said to be universal cohomology classes. We remark that $\Phi([\eta]) \in H^{n, n}(\Gamma \backslash G / K ; \mathbb{C})$.

Let $G^{\prime}:=S O_{0}(2 n, 1)(\subset G)$, and $K^{\prime}:=K \cap G^{\prime} \simeq S O(2 n)$. We assume that $\Gamma^{\prime}:=\Gamma \cap G^{\prime}$ is also a co-compact subgroup of $G^{\prime}$. Then the inclusion $G^{\prime} \hookrightarrow G$ gives rise to a natural map of a totally real, totally geodesic submanifold of dimension $2 n$ into the Kähler manifold $\Gamma \backslash G / K$ of complex dimension $2 n$ :

$$
\iota: Y:=\Gamma^{\prime} \backslash G^{\prime} / K^{\prime} \rightarrow X:=\Gamma \backslash G / K .
$$

We write $[Y]$ for the fundamental class which is a generator of the homology group $H_{2 n}(Y ; \mathbb{Z}) \simeq \mathbb{Z}$. We consider the composition of $\iota_{*}$ and the Poincaré duality map:

$$
H_{2 n}(Y ; \mathbb{C}) \underset{\iota_{*}}{\longrightarrow} H_{2 n}(X ; \mathbb{C}) \underset{P}{\stackrel{\sim}{\longrightarrow}} H_{\text {de } \operatorname{Rham}}^{2 n}(X ; \mathbb{C}) \simeq \bigoplus_{p+q=2 n} H^{p, q}(X ; \mathbb{C}) .
$$

We write

$$
\mathcal{M}(Y):=P \circ \iota_{*}[Y]=\sum_{p+q=2 n} \mathcal{M}^{p, q}(Y) \in \bigoplus_{p+q=2 n} H^{p, q}(X ; \mathbb{C})
$$

corresponding to the Hodge decomposition. Here is our main result in this setting:

Theorem. The $(n, n)$-type Hodge component of the Poincaré dual of the modular symbol $\iota_{*}[Y]$ is given by

$$
\mathcal{M}^{n, n}(Y)=\frac{\operatorname{vol}(Y)}{\operatorname{vol}(X)} \Phi[\eta]
$$

with a natural normalization of $\eta$.

The above theorem is very easy to prove when $n=1$. In fact, in this case $S O_{0}(2,2)$ is a product $S L_{2}(\mathbb{R}) \times S L_{2}(\mathbb{R})$ up to central subgroups, and the cycle derived from $S O_{0}(2,1) \hookrightarrow S O_{0}(2,2)$ is (although itself is not algebraic, but) a "partial complex conjugation" of an algebraic cycle with respect to the complex conjugate on the second factor of $\mathfrak{H} \times \mathfrak{H}$. Therefore the main theorem in this case is 
equivalent to the well-known fact that the Poincaré duals of algebraic 2-cycles on a compact algebraic surface over $\mathbb{C}$ have no $(2,0)+(0,2)$ type Hodge components. The interesting fact is that the similar fact persists in higher dimensional cases!

Here is a brief sketch of the main idea of the proof: We consider the following interactions:

the topology of the embedding of a submanifold $i: N \hookrightarrow M$,

$\downarrow \uparrow$

the pullback of functions (or forms) $\quad i^{*}: \mathcal{E}^{*}(N) \leftarrow \mathcal{E}^{*}(M)$.

We could expect to extract some knowledge of the topology of the embedding $i: N \hookrightarrow M$ from the understanding of the pullback $i^{*}: \mathcal{E}^{*}(N) \leftarrow \mathcal{E}^{*}(M)$. In particular, if $i$ is equivariant with respect to Lie groups $G^{\prime} \hookrightarrow G$, then the pullback $i^{*}$ factors through the restriction of (infinite dimensional) representations of $G$ with respect to $G^{\prime}$. In this way, representation theory gives a clue to the understanding of the topology of the equivariant embedding of a submanifold, although the branching law of a unitary representation is in general a very hard problem for non-compact Lie groups. The idea of our methods in this paper is along this line, to "translate" the information about the restrictions of unitary representations (branching laws) into the topology of modular symbols. The discrete decomposition theorem of $L^{2}(\Gamma \backslash G)$ due to Gel'fand-Piateski-Shapiro [5] made a bridge between the topology of the locally symmetric space $\Gamma \backslash G / K$ and representation theory by the Matsushima-Murakami formula ([3], [14]). Similarly, the recent result on the discrete decomposable restriction of the Zuckerman-Vogan derived functor module $A_{\mathfrak{q}}$ bridges between the topology of the embedding of locally symmetric spaces and representation theory. (We note that what is an equivariant map here is not $\iota: Y=\Gamma^{\prime} \backslash G^{\prime} / K^{\prime} \hookrightarrow X=\Gamma \backslash G / K$ but the induced map between these universal coverings $\widetilde{Y} \hookrightarrow \widetilde{X}$.)

The paper is organized as follows: In $\S 2$ we give an abstract Theorem (Theorem 2.8) which gives a sufficient condition that the modular symbol $\iota_{*}\left[\Gamma^{\prime} \backslash G^{\prime} / K^{\prime}\right]$ is annihilated by the $\pi$-component $H^{\mathrm{d}\left(G^{\prime}\right)}(\pi: \Gamma)\left(\subset H_{\mathrm{de} \text { Rham }}^{\mathrm{d}\left(G^{\prime}\right)}(\Gamma \backslash G / K ; \mathbb{C})\right)$ for $\pi \in \widehat{G}$, where $\mathrm{d}\left(G^{\prime}\right)=\operatorname{dim} G^{\prime} / K^{\prime}$. In $\S 4$ we restate Theorem 2.8 by a very explicit condition in the case where $\left(G, G^{\prime}\right)$ is a semisimple symmetric pair, based on the discrete decomposition theorem (Fact 4.2). This is stated in Theorem 4.3. In $\S 3$, we investigate the invariant part of the modular symbols (Theorem 3.1). In $\S 5$, we apply the results in $\S 2, \S 3$ and $\S 4$ to the case $\left(G, G^{\prime}\right)=\left(S O_{0}(2 n, 2), S O_{0}(2 n, 1)\right)$ and prove the Theorem in this Introduction.

\section{$\S 2$. Vanishing theorem of modular symbols in a primitive form}

In this section, we give a general framework in representation theory that the modular symbol $\iota_{*}\left[\Gamma^{\prime} \backslash G^{\prime} / K^{\prime}\right]$ is annihilated by the $\pi$-component $H^{\mathrm{d}\left(G^{\prime}\right)}(\pi: \Gamma)$ in the de Rham cohomology group $H_{\text {de }}^{*} \operatorname{Rham}(\Gamma \backslash G / K ; \mathbb{C})$ for $\pi \in \widehat{G}$. 
Suppose that $G$ is a real reductive linear Lie group. Let $K$ be a maximal compact subgroup of $G$ and $\Gamma$ a torsion-free, co-compact discrete subgroup of $G$. We assume that $\Gamma \backslash G / K$ is orientable. In particular, this is the case if $G$ is connected.

We say a unitary representation $(\pi, V)$ of $G$ is $G$-admissible if $(\pi, V)$ is decomposed into a discrete Hilbert direct sum of irreducible unitary representations of $G$ with finite multiplicities. The point of the definition is that there is no continuous spectrum in the irreducible decomposition of $G$. A result due to Gel'fandPiateski-Shapiro [5] asserts that the right regular representation on $L^{2}(\Gamma \backslash G)$ is $G$-admissible under the assumption that $\Gamma$ is a co-compact discrete subgroup of $G$. Namely, we have a discrete Hilbert direct sum decomposition:

$$
L^{2}(\Gamma \backslash G) \simeq \sum^{\oplus} \operatorname{Hom}_{G}\left(\pi, L^{2}(\Gamma \backslash G)\right) \otimes \pi=\sum^{\oplus} n_{\Gamma}(\pi) \pi .
$$

Here $\pi$ runs over the unitary dual of $G$ and the multiplicity

$$
n_{\Gamma}(\pi):=\operatorname{dim}_{\mathbb{C}} \operatorname{Hom}_{G}\left(\pi, L^{2}(\Gamma \backslash G)\right)<\infty .
$$

Let $\mathfrak{g}_{0}=\mathfrak{k}_{0}+\mathfrak{p}_{0}$ be a Cartan decomposition of the Lie algebra $\mathfrak{g}_{0}$ of $G$ and $\mathfrak{g}=\mathfrak{k}+\mathfrak{p}$ the complexifications. Let $\mathcal{E}^{p}(\Gamma \backslash G / K)$ be the space of $p$-forms on $\Gamma \backslash G / K$. Because the cotangent bundle $T^{*}(\Gamma \backslash G / K)$ is isomorphic to the bundle $\Gamma \backslash G \underset{K}{\times} \mathfrak{p}^{*} \rightarrow \Gamma \backslash G / K$, which is associated to the principal $K$-bundle $K \rightarrow \Gamma \backslash G \rightarrow$ $\Gamma \backslash G / K$ and the $K$-representation $K \rightarrow G L_{\mathbb{R}}\left(\mathfrak{p}^{*}\right)$, we have

$$
\mathcal{E}^{p}(\Gamma \backslash G / K) \simeq\left(C^{\infty}(\Gamma \backslash G) \otimes \wedge^{p} \mathfrak{p}^{*}\right)^{K} \simeq \operatorname{Hom}_{K}\left(\wedge^{p} \mathfrak{p}, C^{\infty}(\Gamma \backslash G)\right) \quad(p \in \mathbb{N}) .
$$

We fix a non-degenerate symmetric bilinear form $B$ on $\mathfrak{g}_{0}$ such that $B \mid \mathfrak{k}_{0} \times \mathfrak{k}_{0}$ is negative definite, $\left.B\right|_{\mathfrak{p}_{0} \times \mathfrak{p}_{0}}$ is positive definite and that $\mathfrak{k}_{0}$ and $\mathfrak{p}_{0}$ are orthogonal to each other. Then the positive definite bilinear form $\left.B\right|_{\mathfrak{p}_{0} \times \mathfrak{p}_{0}}$ induces a $G$-invariant Riemannian metric on $G / K$, which makes $G / K$ into a Riemannian symmetric space. Through the covering

$$
\varpi: G / K \rightarrow \Gamma \backslash G / K
$$

the Clifford-Klein form $\Gamma \backslash G / K$ is equipped with a Riemannian metric so that $\varpi$ is isometric. We write $\Delta=d d^{*}+d^{*} d$ for the corresponding Laplace-Beltrami operator acting on the space of forms on $\Gamma \backslash G / K$. The space of harmonic $p$-forms is given by

$$
\mathcal{H}_{\Delta}^{p}(\Gamma \backslash G / K):=\left\{\omega \in \mathcal{E}^{p}(\Gamma \backslash G / K): \Delta \omega=0\right\} .
$$

Then the Hodge theory asserts a natural isomorphism

$$
H_{\text {de Rham }}^{*}(\Gamma \backslash G / K ; \mathbb{C}) \simeq \mathcal{H}_{\Delta}^{*}(\Gamma \backslash G / K) .
$$


Let $\left(\pi, V_{\pi}\right)$ be an irreducible $(\mathfrak{g}, K)$-module. Using the identification

$$
\mathcal{E}^{*}(\Gamma \backslash G / K) \simeq\left(C^{\infty}(\Gamma \backslash G) \otimes \wedge^{*} \mathfrak{p}^{*}\right)^{K} \simeq \operatorname{Hom}_{K}\left(\wedge^{*} \mathfrak{p}, C^{\infty}(\Gamma \backslash G)\right),
$$

we define a linear map

$$
T_{\pi}: \operatorname{Hom}_{K}\left(\wedge^{*} \mathfrak{p}, \pi\right) \otimes \operatorname{Hom}_{\mathfrak{g}, K}\left(\pi, C^{\infty}(\Gamma \backslash G)\right) \rightarrow \mathcal{E}^{*}(\Gamma \backslash G / K), \quad \psi \otimes \varphi \mapsto \varphi \circ \psi .
$$

Let $\widehat{G}$ be the set of the equivalence classes of irreducible $(\mathfrak{g}, K)$-modules that are unitarizable. We note that $\widehat{G}$ is naturally identified with the unitary dual of $G$ (Harish-Chandra). Let $U(\mathfrak{g})$ be the enveloping algebra of the complexified Lie algebra $\mathfrak{g}=\mathfrak{g}_{0} \otimes_{\mathbb{R}} \mathbb{C}, Z(\mathfrak{g})$ the center of $U(\mathfrak{g})$, and $c_{G} \in Z(\mathfrak{g})$ the Casimir element defined by the non-degenerate symmetric bilinear form $B$. We define a subset $\widehat{G}_{0}(\subset \widehat{G})$ by

$$
\widehat{G}_{0}:=\left\{\pi \in \widehat{G}: \pi\left(c_{G}\right)=0\right\} .
$$

Here, we have used the same notation $\pi$ for the representation of $U(\mathfrak{g})$.

The left action of $G$ on $G / K$ induces the representation of $U(\mathfrak{g})$ on the space of forms on $G / K$. In particular, the Casimir element $c_{G}(\in Z(\mathfrak{g}) \subset U(\mathfrak{g}))$ acts on $\mathcal{E}^{*}(G / K)$ as the Laplace-Beltrami operator, since the Riemannian metric on $G / K$ is induced from $\left.B\right|_{\mathfrak{p}_{0} \times \mathfrak{p}_{0}}$. Because the quotient map $\varpi: G / K \rightarrow \Gamma \backslash G / K$ is a Riemannian covering, $c_{G}$ induces the Laplace-Beltrami operator $\Delta$ on $\Gamma \backslash G / K$. Therefore, we have

$$
\text { Image } T_{\pi} \subset \mathcal{H}_{\Delta}^{*}(\Gamma \backslash G / K) \simeq H_{\text {de Rham }}^{*}(\Gamma \backslash G / K ; \mathbb{C})
$$

if and only if $\pi \in \widehat{G}_{0}$. We say that the subspace of $H_{\text {de Rham }}^{*}(\Gamma \backslash G / K$; $)$ corresponding to Image $T_{\pi}$ is the $\pi$-component, and write $H^{*}(\pi: \Gamma)$. That is,

$$
H^{p}(\pi: \Gamma):=\operatorname{Image} T_{\pi} \cap H_{\text {de Rham }}^{p}(\Gamma \backslash G / K ; \mathbb{C}) \quad(p \in \mathbb{N}),
$$

via the isomorphism (2.3.1). Then the Matsushima-Murakami formula and a result of Borel-Wallach are summarized as:

Lemma 2.4 ([3], [14]). Retain the above setting. We have

$$
\begin{aligned}
H^{*}(\pi: \Gamma) & \simeq n_{\Gamma}(\pi) H^{*}(\mathfrak{g}, K ; \pi) . \\
H_{\text {de Rham }}^{*}(\Gamma \backslash G / K ; \mathbb{C}) & =\bigoplus_{\pi \in \widehat{G}_{0}} H^{*}(\pi: \Gamma) . \\
H^{\operatorname{dim} G / K}(\pi: \Gamma) & \neq 0 \quad \text { if and only if } \pi=\mathbf{1 .}
\end{aligned}
$$

Here is an easy application of the Hodge theory.

Lemma 2.5. Suppose $\omega \in \mathcal{E}^{*}(\Gamma \backslash G / K)$ is a closed form such that

$$
\left.\omega=\sum_{i} T_{\pi_{i}}\left(\delta_{i}\right) \quad \text { (finite sum }\right)
$$


where $\pi_{i}$ 's are irreducible $(\mathfrak{g}, K)$-modules and

$$
\delta_{i} \in \operatorname{Hom}_{K}\left(\wedge^{*} \mathfrak{p}, \pi_{i}\right) \otimes \operatorname{Hom}_{\mathfrak{g}, K}\left(\pi_{i}, C^{\infty}(\Gamma \backslash G)\right) .
$$

We define a harmonic form by

$$
\omega_{0}:=\sum_{\pi_{i} \in \widehat{G}_{0}} T_{\pi_{i}}\left(\delta_{i}\right)
$$

Then, passing to the cohomology group $H_{\mathrm{de}}^{*} \mathrm{Rham}(\Gamma \backslash G / K)$, we have

$$
[\omega]=\left[\omega_{0}\right] .
$$

Proof. Because $\pi_{i}$ is an irreducible $(\mathfrak{g}, K)$-module, the Casimir element $c_{G}$ acts by a scalar, say $\lambda_{i} \in \mathbb{C}$, on the representation space of each $\pi_{i}$ by Schur's lemma, so that

$$
\text { Image } T_{\pi_{i}} \subset\left\{\omega \in \mathcal{E}^{*}(\Gamma \backslash G / K): \Delta \omega=\lambda_{i} \omega\right\} \text {. }
$$

We set

$$
\omega_{1}:=\sum_{\pi_{i} \in \widehat{G} \backslash \widehat{G}_{0}} T_{\pi_{i}}\left(\delta_{i}\right)
$$

Then we have $\omega=\omega_{0}+\omega_{1}$. Since $d \omega=0$ and $\Delta \omega_{0}=0$, we have $d \omega_{1}=d \omega-d \omega_{0}=$ 0 .

Since $\lambda_{i} \neq 0$ for each $\pi_{i} \in \widehat{G} \backslash \widehat{G}_{0}, \omega_{1} \in \operatorname{Image}\left(\Delta: \mathcal{E}^{*}(\Gamma \backslash G / K) \rightarrow \mathcal{E}^{*}(\Gamma \backslash G / K)\right)$. Denote by $G$ the Green's operator. Then we have

$$
\omega_{1}=\Delta G \omega_{1}=d d^{*} G \omega_{1}+d^{*} d G \omega_{1} .
$$

Since $G$ commutes with $d$, we have

$$
\omega_{1}=d d^{*} G \omega_{1}+d^{*} G d \omega_{1}
$$

Because $\omega_{1}$ is a closed form, we have

$$
\omega_{1}=d d^{*} G \omega_{1} .
$$

Hence $\omega_{1}$ is an exact form. Therefore, $[\omega]=\left[\omega_{0}+\omega_{1}\right]=\left[\omega_{0}\right]$.

Let $G^{\prime}$ be a closed subgroup that is reductive in $G$. Assume that $\Gamma^{\prime}:=\Gamma \cap G^{\prime}$ is also a co-compact subgroup of $G^{\prime}$. We take a maximal compact subgroup $K$ of $G$ such that $K^{\prime}:=K \cap G^{\prime}$ is a maximal compact subgroup of $G^{\prime}$. For simplicity, we put $Y=\Gamma^{\prime} \backslash G^{\prime} / K^{\prime}$ and $X=\Gamma \backslash G / K$. Then we have natural maps $i$ and $\iota$ : 


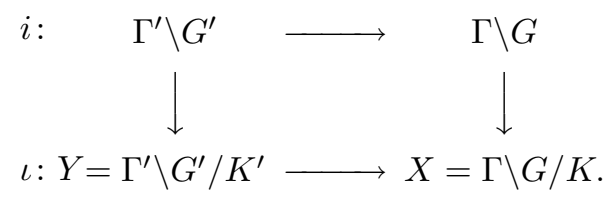

Assume moreover that $Y$ is orientable (this is always satisfied if $G^{\prime}$ is connected). We write $[Y]$ for the fundamental class which is a generator of the homology group $H_{\mathrm{d}\left(G^{\prime}\right)}(Y ; \mathbb{Z}) \simeq \mathbb{Z}$. Here, $\mathrm{d}\left(G^{\prime}\right):=\operatorname{dim} Y=\operatorname{dim} G^{\prime} / K^{\prime}$. The homology class

$$
\iota_{*}([Y]) \in H_{\mathrm{d}\left(G^{\prime}\right)}(X ; \mathbb{Z})
$$

is said to be the (generalized) modular symbol.

The investigation of the induced homomorphism $\iota_{*}: H_{*}(Y ; \mathbb{C}) \rightarrow H_{*}(X ; \mathbb{C})$ is equivalent to that of the pullback homomorphism

$$
\iota^{*}: H_{\mathrm{de} \operatorname{Rham}}^{*}(X ; \mathbb{C}) \rightarrow H_{\mathrm{de} \operatorname{Rham}}^{*}(Y ; \mathbb{C}) .
$$

To study the latter object, we recall the notion of the discrete decomposable restriction introduced in [6], [7], [8].

Definition 2.7 ([8], Definition 1.1, 1.2). We say that an irreducible unitarizable $(\mathfrak{g}, K)$-module $\left(\pi, V_{\pi}\right)$ is discretely decomposable, if $\pi$ is isomorphic as $\left(\mathfrak{g}^{\prime}, K^{\prime}\right)$ modules to an algebraic discrete sum of irreducible $\left(\mathfrak{g}^{\prime}, K^{\prime}\right)$-modules:

$$
\pi \simeq \bigoplus_{\tau \in \widehat{G}^{\prime}} m_{\pi}(\tau) \tau_{K^{\prime}}
$$

where $m_{\pi}(\tau) \in \mathbb{N} \cup\{\infty\}$.

Let $\bar{\pi}$ be the unitary representation defined on the Hilbert completion $\overline{V_{\pi}}$. We allow us to use the terminology that the restriction $\left.\pi\right|_{K^{\prime}}$ is $K^{\prime}$-admissible for a compact subgroup $K^{\prime}(\subset K)$, if the restriction $\left.\bar{\pi}\right|_{K^{\prime}}$ is $K^{\prime}$-admissible, equivalently if $\operatorname{dim} \operatorname{Hom}_{K^{\prime}}(\mu, \pi)=\operatorname{dim} \operatorname{Hom}_{K^{\prime}}(\mu, \bar{\pi})<\infty$ for any $\mu \in \widehat{K^{\prime}}$ (we recall that $K^{\prime}$ is compact). Then if the restriction $\left.\pi\right|_{K^{\prime}}$ is $K^{\prime}$-admissible, then $\pi$ is discretely decomposable as a $\left(\mathfrak{g}^{\prime}, K^{\prime}\right)$-module and $m_{\pi}(\tau)<\infty$ for any $\tau \in \widehat{G^{\prime}}$ (see [8], Proposition 1.6(2)).

Theorem 2.8. Suppose we are in the above setting. We assume the following three conditions.

i) $\pi \in \widehat{G}$ is infinite dimensional.

ii) The restriction $\left.\pi\right|_{K^{\prime}}$ is $K^{\prime}$-admissible.

iii) $G^{\prime}$ is semisimple without compact factors. 
Consider the natural perfect pairing $\langle$,$\rangle given by$

$$
\langle,\rangle: H_{\text {de } \operatorname{Rham}}^{j}(X ; \mathbb{C}) \times H_{j}(X ; \mathbb{C}) \rightarrow \mathbb{C} .
$$

Then the modular symbol $\iota_{*}[Y] \in H_{\mathrm{d}\left(G^{\prime}\right)}(X ; \mathbb{C})$ is annihilated by $H^{\mathrm{d}\left(G^{\prime}\right)}(\pi: \Gamma)$ $\left(\subset H_{\text {de Rham }}^{\mathrm{d}\left(G^{\prime}\right)}(X ; \mathbb{C})\right)$, where $\mathrm{d}\left(G^{\prime}\right)=\operatorname{dim} Y=\operatorname{dim} \mathfrak{p}_{0}^{\prime}$.

Proof. In view of the isomorphisms (2.1)

$$
\begin{aligned}
& \mathcal{E}^{*}(X) \simeq \operatorname{Hom}_{K}\left(\wedge^{*} \mathfrak{p}, C^{\infty}(\Gamma \backslash G)\right), \\
& \mathcal{E}^{*}(Y) \simeq \operatorname{Hom}_{K^{\prime}}\left(\wedge^{*} \mathfrak{p}^{\prime}, C^{\infty}\left(\Gamma^{\prime} \backslash G^{\prime}\right)\right),
\end{aligned}
$$

the pullback $\iota^{*}: \mathcal{E}^{*}(X) \rightarrow \mathcal{E}^{*}(Y)$ is identified with

$$
\operatorname{Hom}_{K}\left(\wedge^{*} \mathfrak{p}, C^{\infty}(\Gamma \backslash G)\right) \rightarrow \operatorname{Hom}_{K^{\prime}}\left(\wedge^{*} \mathfrak{p}^{\prime}, C^{\infty}\left(\Gamma^{\prime} \backslash G^{\prime}\right)\right), \delta \mapsto i^{*} \circ \delta \circ j
$$

Here,

$$
j: \wedge^{*} \mathfrak{p}^{\prime} \rightarrow \wedge^{*} \mathfrak{p}
$$

is an injective $K^{\prime}$-homomorphism induced from $\mathfrak{p}^{\prime} \hookrightarrow \mathfrak{p}$, and

$$
i^{*}: C^{\infty}(\Gamma \backslash G) \rightarrow C^{\infty}\left(\Gamma^{\prime} \backslash G^{\prime}\right)
$$

is the pullback $C^{\infty}$-functions with respect to the embedding $i: \Gamma^{\prime} \backslash G^{\prime} \hookrightarrow \Gamma \backslash G$. Suppose an irreducible $(\mathfrak{g}, K)$-module $\left(\pi, V_{\pi}\right)$ is discretely decomposable as a $\left(\mathfrak{g}^{\prime}, K^{\prime}\right)$ module. Namely, $\pi$ is isomorphic to an algebraic direct sum

$$
\pi \simeq \bigoplus_{\tau \in \widehat{G}^{\prime}} \underbrace{\tau \oplus \cdots \oplus \tau}_{m_{\pi}(\tau)}
$$

as a $\left(\mathfrak{g}^{\prime}, K^{\prime}\right)$-module. For each $\tau \in \widehat{G^{\prime}}$ and each $k\left(1 \leq k \leq m_{\pi}(\tau)\right)$, let denote by

$$
\operatorname{pr}_{\tau}^{(k)}: \pi \rightarrow \tau
$$

the projection onto the $k$-th component of $\tau$ in the direct sum (2.8.1), and by

$$
e_{\tau}^{(k)}: \tau \rightarrow \pi
$$

the injection into the $k$-th component of $\tau$. Then both $\operatorname{pr}_{\tau}^{(k)}$ and $e_{\tau}^{(k)}$ are $\left(\mathfrak{g}^{\prime}, K^{\prime}\right)$ homomorphisms. We note that if $U \subset V_{\pi}$ is a $K$-stable finite dimensional subspace, then $\operatorname{pr}_{\tau}^{(k)}(U)=0$ except for finitely many $\tau \in \widehat{G^{\prime}}([8]$, Proposition 1.6(1)). In particular, if $\psi \in \operatorname{Hom}_{K}\left(\wedge^{*} \mathfrak{p}, V_{\pi}\right)$, then the right side of

$$
\psi=\sum_{\tau \in \widehat{G}^{\prime}} \sum_{k=1}^{m_{\pi}(\tau)} e_{\tau}^{(k)} \circ \operatorname{pr}_{\tau}^{(k)} \circ \psi
$$


is actually a finite sum because $\operatorname{dim} \wedge^{*} \mathfrak{p}<\infty$. If $\varphi \in \operatorname{Hom}_{\mathfrak{g}, K}\left(V_{\pi}, C^{\infty}(\Gamma \backslash G)\right)$, then we have

$$
i^{*} \circ \varphi \circ \psi \circ j=\sum_{\tau \in \widehat{G}^{\prime}} \sum_{k=1}^{m_{\pi}(\tau)}\left(i^{*} \circ \varphi \circ e_{\tau}^{(k)}\right) \circ\left(\operatorname{pr}_{\tau}^{(k)} \circ \psi \circ j\right) .
$$

We note $\operatorname{pr}_{\tau}^{(k)} \circ \psi \circ j \in \operatorname{Hom}_{K^{\prime}}\left(\wedge^{*} \mathfrak{p}^{\prime}, \tau\right)$ and $i^{*} \circ \varphi \circ e_{\tau}^{(k)} \in \operatorname{Hom}_{\mathfrak{g}^{\prime}, K^{\prime}}\left(\tau, C^{\infty}\left(\Gamma^{\prime} \backslash G^{\prime}\right)\right)$. Thus,

$$
\left(i^{*} \circ \varphi \circ e_{\tau}^{(k)}\right) \circ\left(\operatorname{pr}_{\tau}^{(k)} \circ \psi \circ j\right) \in \operatorname{Image} T_{\tau}^{\prime}\left(\subset \mathcal{E}^{*}\left(\Gamma^{\prime} \backslash G^{\prime} / K^{\prime}\right)\right) .
$$

Here, we have used a notation similar to (2.2):

$$
T_{\tau}^{\prime}: \operatorname{Hom}_{K^{\prime}}\left(\wedge^{*} \mathfrak{p}^{\prime}, \tau\right) \otimes \operatorname{Hom}_{\mathfrak{g}^{\prime}, K^{\prime}}\left(\tau, C^{\infty}\left(\Gamma^{\prime} \backslash G^{\prime}\right)\right) \rightarrow \mathcal{E}^{*}\left(\Gamma^{\prime} \backslash G^{\prime} / K^{\prime}\right)=\mathcal{E}^{*}(Y),
$$

for each irreducible $\left(\mathfrak{g}^{\prime}, K^{\prime}\right)$-module $\tau$.

Because the sum (2.8.2) is a finite sum, we have

$$
\left[i^{*} \circ \varphi \circ \psi \circ j\right]=\sum_{\tau \in \widehat{G}_{0}^{\prime}} \sum_{k=1}^{m_{\pi}(\tau)}\left[\left(i^{*} \circ \varphi \circ e_{\tau}^{(k)}\right) \circ\left(\operatorname{pr}_{\tau}^{(k)} \circ \psi \circ j\right)\right] \in H_{\text {de } \operatorname{Rham}}^{*}(Y ; \mathbb{C}),
$$

from Lemma 2.5. Since the cohomology group of the top degree $\mathrm{d}\left(G^{\prime}\right)=\operatorname{dim} G^{\prime} / K^{\prime}$, namely,

$$
H^{\mathrm{d}\left(G^{\prime}\right)}\left(\tau: \Gamma^{\prime}\right)=\text { Image } T_{\tau}^{\prime} \cap H_{\mathrm{de} \operatorname{Rham}}^{\mathrm{d}\left(G^{\prime}\right)}(Y ; \mathbb{C}),
$$

is non-zero if and only if $\tau=\mathbf{1}$ (Lemma 2.4(3)), we have

$$
\left\langle\left[i^{*} \circ \varphi \circ \psi \circ j\right],[Y]\right\rangle=\sum_{k=1}^{m_{\pi}(\mathbf{1})}\left\langle\left[\left(i^{*} \circ \varphi \circ e_{\mathbf{1}}^{(k)}\right) \circ\left(\operatorname{pr}_{\mathbf{1}}^{(k)} \circ \psi \circ j\right)\right],[Y]\right\rangle
$$

with respect to the natural pairing $H_{\mathrm{de} R h a m}^{\mathrm{d}\left(G^{\prime}\right)}(Y ; \mathbb{C}) \times H_{\mathrm{d}\left(G^{\prime}\right)}(Y ; \mathbb{C}) \rightarrow \mathbb{C}$.

In order to prove the right side of $(2.8 .3)$ to be zero, it suffices to show $m_{\pi}(\mathbf{1})=0$. Namely, $\left.\pi\right|_{G^{\prime}}$ does not contain the trivial representation as a direct summand. If it were not the case, then any irreducible unitary representation of $G^{\prime}$ arising in the decomposition of the restriction $\left.\pi\right|_{G^{\prime}}$ would be finite dimensional, because any irreducible summands in the restriction $\left.\bar{\pi}\right|_{G^{\prime}}$ has the same associated variety (see [9], Part II, Theorem 3.5; see also [8], Corollary 3.9). On the other hand, if $G^{\prime}$ is semisimple without compact factors then the number of equivalence classes of irreducible unitary finite dimensional representations of $G^{\prime}$ is at most that of connected components of $G^{\prime}$, which is finite. This means that the restriction $\left.\pi\right|_{G^{\prime}}$ is decomposed into finitely many, irreducible, finite dimensional representations of $G^{\prime}$ with finite multiplicities, which is absurd because $\pi$ is infinite 
dimensional. Therefore we conclude that the restriction $\left.\pi\right|_{G^{\prime}}$ does not contain the trivial representation of $G^{\prime}$ as a direct summand, namely, $m_{\pi}(\mathbf{1})=0$. Hence we have proved Theorem 2.8 .

Similarly to Theorem 2.8, we formulate a vanishing theorem about the cup products in the de Rham cohomology ring $H_{\text {de Rham }}^{*}(\Gamma \backslash G / K ; \mathbb{C})$.

Theorem 2.9. Suppose that $G$ is a connected semisimple linear Lie group without compact factors and that $\Gamma$ is a torsion-free co-compact discrete subgroup of $G$. Let $\pi, \pi^{\prime} \in \widehat{G}$. Assume the following two conditions.

i) At least one of $\pi$ or $\pi^{\prime}$ is infinite dimensional.

ii) The tensor product $\pi \otimes \pi^{\prime}$ is $K$-admissible.

Then the cup product $\alpha \cup \alpha^{\prime}$ is zero in $H_{\mathrm{de} \mathrm{Rham}}^{\mathrm{d}(G)}(\Gamma \backslash G / K ; \mathbb{C})$ for any $\alpha \in H^{i}(\pi: \Gamma)$ and $\alpha^{\prime} \in H^{\mathrm{d}(G)-i}\left(\pi^{\prime}: \Gamma\right)$.

Proof. Let $\alpha \in H_{\text {de Rham }}^{i}(\Gamma \backslash G / K ; \mathbb{C})$ and $\alpha^{\prime} \in H_{\text {de Rham }}^{\mathrm{d}(G)-i}(\Gamma \backslash G / K ; \mathbb{C})$. The cup product $\alpha \cup \alpha^{\prime}$ is given by the pullback of the cohomology class with respect to the diagonally embedding map

$$
\iota: G \rightarrow G \times G, g \mapsto(g, g),
$$

namely, we have $\iota^{*}\left(\alpha \wedge \alpha^{\prime}\right)=\alpha \cup \alpha^{\prime}$, where

$$
\iota^{*}: H_{\mathrm{de} \mathrm{Rham}}^{i+(\mathrm{d}(G)-i)}((\Gamma \times \Gamma) \backslash(G \times G) /(K \times K) ; \mathbb{C}) \rightarrow H_{\mathrm{de} \mathrm{Rham}}^{\mathrm{d}(G)}(\Gamma \backslash G / K ; \mathbb{C}) .
$$

We note that $\alpha \wedge \alpha^{\prime}$ belongs to the $\pi \otimes \pi^{\prime}$ components of the cohomology group $H_{\text {de Rham }}^{\mathrm{d}(G)}((\Gamma \times \Gamma) \backslash(G \times G) /(K \times K) ; \mathbb{C})$ and that the tensor product $\pi \otimes \pi^{\prime}$ is nothing but the restriction of $\pi \otimes \pi^{\prime}$ with respect to $\operatorname{diag}(\mathfrak{g}, K)$, which is diagonally embedded into $(\mathfrak{g}+\mathfrak{g}, K \times K)$. Applying Theorem 2.8 with the pair $(G \times G, G)$, we see that $\iota_{*}[\Gamma \backslash G / K]$ is annihilated by the $\pi \otimes \pi^{\prime}$ components of the de Rham cohomology group $H_{\mathrm{de} \text { Rham }}^{\mathrm{d}(G)}((\Gamma \times \Gamma) \backslash(G \times G) /(K \times K) ; \mathbb{C})$. Hence, we have

$$
\int_{\Gamma \backslash G / K} \alpha \cup \alpha^{\prime}=\int_{\Gamma \backslash G / K} \iota^{*}\left(\alpha \wedge \alpha^{\prime}\right)=\int_{\iota_{*}(\Gamma \backslash G / K)} \alpha \wedge \alpha^{\prime}=0 .
$$

Since $G$ is connected, $\Gamma \backslash G / K$ is orientable. Therefore $\alpha \cup \alpha^{\prime}=0$.

Applying Theorem 2.9 to $\pi^{\prime}=\mathbf{1}$, the trivial representation of $G$, we have:

Corollary 2.10. Retain the setting of Theorem 2.9. Then

$$
H^{i}(\mathbf{1}: \Gamma) \quad\left(\subset H_{\text {de }}^{i} \operatorname{Rham}(\Gamma \backslash G / K ; \mathbb{C})\right)
$$




$$
\bigoplus_{\substack{\pi \in \widehat{G}_{0} \\ \pi \neq 1}} H^{\mathrm{d}(G)-i}(\pi: \Gamma) \quad\left(\subset H_{\mathrm{de} \operatorname{Rham}}^{\mathrm{d}(G)-i}(\Gamma \backslash G / K ; \mathbb{C})\right)
$$

are the annihilators to each other in the perfect pairing

$$
H_{\text {de Rham }}^{i}(\Gamma \backslash G / K ; \mathbb{C}) \times H_{\text {de Rham }}^{\mathrm{d}(G)-i}(\Gamma \backslash G / K ; \mathbb{C}) \rightarrow \mathbb{C}, \quad \alpha, \beta \mapsto \int_{\Gamma \backslash G / K} \alpha \cup \beta .
$$

We note that the restriction of (2.10.1) to the pairing

$$
H^{i}(\mathbf{1}: \Gamma) \times H^{\mathrm{d}(G)-i}(\mathbf{1}: \Gamma) \rightarrow \mathbb{C}
$$

is non-degenerate (cf. Lemma 3.2). Corollary 2.10 will be used in the proof of Theorem 3.1 that gives a comparison of the modular symbols with the corresponding objects for compact homogeneous manifolds.

\section{$\S 3$. The invariant part of modular symbols}

In this section we examine the invariant part (the 1-component) of modular symbols.

We recall the setting in $\S 2$. That is, $G$ is a connected real reductive linear Lie group, $\theta$ is a Cartan involution, $\mathfrak{g}_{0}=\mathfrak{k}_{0}+\mathfrak{p}_{0}$ is a Cartan decomposition, $K=G^{\theta}$ is a maximal compact subgroup of $G$, and $\Gamma$ is a torsion-free co-compact subgroup. Suppose $G^{\prime}$ is a connected closed subgroup which is $\theta$-stable such that $\Gamma^{\prime}=G^{\prime} \cap \Gamma$ is co-compact in $G^{\prime}$.

We assume that $G$ is contained in a complex Lie group $G_{\mathbb{C}}$ with Lie algebra $\mathfrak{g} \simeq \mathfrak{g}_{0} \otimes \mathbb{C}$. Let $G_{U}$ and $G_{U}^{\prime}$ be the analytic subgroups of $G_{\mathbb{C}}$ with Lie algebras $\mathfrak{k}_{0}+\sqrt{-1} \mathfrak{p}_{0}$ and $\mathfrak{k}_{0}^{\prime}+\sqrt{-1} \mathfrak{p}_{0}^{\prime}$, respectively. Then both $G_{U}$ and $G_{U}^{\prime}$ are compact. We write $Y_{U}:=G_{U}^{\prime} / K^{\prime}, Y:=\Gamma^{\prime} \backslash G^{\prime} / K^{\prime}, X_{U}:=G_{U} / K$ and $X:=\Gamma \backslash G / K$. Let $\iota_{U}: Y_{U} \hookrightarrow X_{U}$ and $\iota: Y \hookrightarrow X$ be the natural embeddings. The Poincaré dual of the modular symbol, denoted by $\mathcal{M}(Y)$, is by definition the image of the fundamental class $[Y]$ of the composition map

$$
H_{\operatorname{dim} Y}(Y ; \mathbb{C}) \underset{\iota_{*}}{\rightarrow} H_{\operatorname{dim} Y}(X ; \mathbb{C}) \underset{P}{\stackrel{\sim}{\longrightarrow}} H_{\operatorname{de} \operatorname{Rham}}^{\operatorname{codim} Y}(X ; \mathbb{C}) .
$$

Similarly, we define $\mathcal{M}\left(Y_{U}\right) \in H_{\text {de } \operatorname{Rham}}^{\operatorname{codim} Y}(X ; \mathbb{C})$ to be the image of the fundamental class $\left[Y_{U}\right]$ of the composition map

$$
H_{\operatorname{dim} Y}\left(Y_{U} ; \mathbb{C}\right) \underset{\iota_{U *}}{\longrightarrow} H_{\operatorname{dim} Y}\left(X_{U} ; \mathbb{C}\right) \underset{P_{U}}{\stackrel{\sim}{\longrightarrow}} H_{\operatorname{de} \operatorname{Rham}}^{\operatorname{codim} Y}\left(X_{U} ; \mathbb{C}\right),
$$

where $P$ and $P_{U}$ are the Poincaré duality maps. Here we note that $\operatorname{dim} Y_{U}=\operatorname{dim} Y$ and $\operatorname{dim} X_{U}=\operatorname{dim} X$. 
We denote by

$$
\operatorname{pr}_{\pi}: H_{\text {de Rham }}^{*}(X ; \mathbb{C}) \rightarrow H^{*}(\pi: \Gamma)
$$

for the projection onto the $\pi$-component, corresponding to the direct sum decomposition (see Lemma 2.4)

$$
H_{\text {de } \operatorname{Rham}}^{*}(X ; \mathbb{C})=\bigoplus_{\pi \in \widehat{G}_{0}} H^{*}(\pi: \Gamma) .
$$

The $\pi$-component corresponding to $\pi=\mathbf{1}$ (the trivial representation), namely,

$$
H^{*}(\mathfrak{g}, K ; \mathbb{C})=H^{*}(\mathbf{1}: \Gamma) \subset H_{\text {de Rham }}^{*}(\Gamma \backslash G / K ; \mathbb{C})
$$

is interpreted geometrically by using the diagram:

$$
X_{U}=G_{U} / K \hookrightarrow G_{\mathbb{C}} / K_{\mathbb{C}} \hookleftarrow G / K \rightarrow \Gamma \backslash G / K=X,
$$

as follows; start with a $G_{U}$-invariant representative of the de Rham cohomology

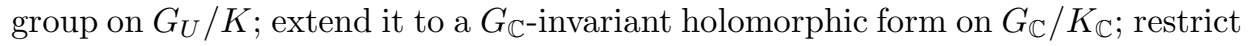
it to $G / K$; and push down to a form on $\Gamma \backslash G / K$ (this is possible because of the invariance by $G$, especially by $\Gamma$ ). Then we have defined a linear map

$$
\Psi: H_{\mathrm{de}}^{*} \operatorname{Rham}\left(X_{U} ; \mathbb{C}\right) \rightarrow H_{\mathrm{de}}^{*} \operatorname{Rham}(X ; \mathbb{C}) .
$$

Similarly, we define

$$
\Psi^{\prime}: H_{\text {de } \operatorname{Rham}}^{*}\left(Y_{U} ; \mathbb{C}\right) \rightarrow H_{\text {de } \operatorname{Rham}}^{*}(Y ; \mathbb{C})
$$

Theorem 3.1. With notation as above, we have

$$
\operatorname{pr}_{1}(\mathcal{M}(Y))=\frac{\operatorname{vol}(Y)}{\operatorname{vol}(X)} \Psi\left(\mathcal{M}\left(Y_{U}\right)\right)
$$

Before proving Theorem 3.1, we recall:

\section{Lemma 3.2.}

1) $\Psi$ is a well-defined, injective ring homomorphism with image $H^{*}(\mathbf{1}: \Gamma)$.

2) $\Psi$ sends characteristic classes on $X_{U}$ to the corresponding characteristic classes on $X$.

3) $\int_{X} \Psi(\omega)=\operatorname{vol}(X) \int_{X_{U}} \omega$ for any $\omega \in H_{\mathrm{de}}^{\operatorname{dim} X} X\left(X_{U} ; \mathbb{C}\right)$.

4) The following diagram commutes: 


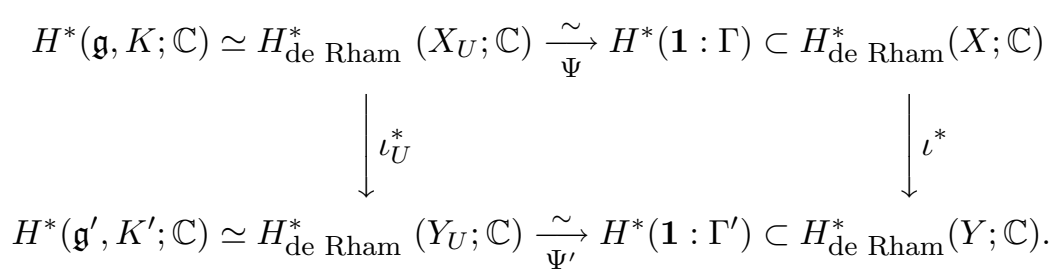

Proof of Lemma 3.2. See the main theorem in [10] (treated in a more general setting than here).

Proof of Theorem 3.1. We fix an arbitrary $\alpha \in H_{\mathrm{de}}^{\operatorname{dim} Y} \operatorname{Rham}\left(X_{U} ; \mathbb{C}\right)$. Then

$$
\begin{array}{rlrl}
\int_{X} \mathcal{M}(Y) \wedge \Psi(\alpha) & =\int_{X} P \circ \iota_{*}[Y] \wedge \Psi(\alpha) & & \\
& =\int_{\iota_{*}[Y]} \Psi(\alpha) & & \text { (Poincaré duality) } \\
& =\int_{[Y]} \iota^{*} \circ \Psi(\alpha) & \\
& =\int_{[Y]} \Psi^{\prime} \circ \iota_{U}^{*} \alpha & & \text { (Lemma 3.2 (4)) } \\
& =\operatorname{vol}(Y) \int_{Y_{U}} \iota_{U}^{*} \alpha & & \text { (Lemma 3.2 }(3))
\end{array}
$$

Similarly, we have

$$
\begin{array}{rlrl}
\int_{X} \Psi\left(\mathcal{M}\left(Y_{U}\right)\right) \wedge \Psi(\alpha) & =\int_{X} \Psi\left(\mathcal{M}\left(Y_{U}\right) \wedge \alpha\right) & \quad \text { (Lemma 3.2 (1)) } \\
& =\operatorname{vol}(X) \int_{\iota_{U *}\left[Y_{U}\right]} \alpha \quad \text { (Lemma 3.2 (3)) } \\
& =\operatorname{vol}(X) \int_{Y_{U}} \iota_{U}^{*} \alpha .
\end{array}
$$

These two formulae mean that

$$
\mathcal{M}(Y)-\Psi\left(\frac{\operatorname{vol}(Y)}{\operatorname{vol}(X)} \mathcal{M}\left(Y_{U}\right)\right) \in H_{\text {de Rham }}^{\operatorname{codim} Y}(X ; \mathbb{C})
$$

is annihilated by

$$
\operatorname{Image}\left(\Psi: H_{\operatorname{de}}^{\operatorname{dim} Y} \operatorname{Rham}\left(X_{U} ; \mathbb{C}\right) \rightarrow H_{\operatorname{de}}^{\operatorname{dim} Y} \operatorname{Rham}(X ; \mathbb{C})\right)=H^{\operatorname{dim} Y}(\mathbf{1}: \Gamma) .
$$


It follows from Corollary 2.10 that

$$
\mathcal{M}(Y)-\frac{\operatorname{vol}(Y)}{\operatorname{vol}(X)} \Psi\left(\mathcal{M}\left(Y_{U}\right)\right) \in \bigoplus_{\substack{\pi \in \widehat{G}_{0} \\ \pi \neq 1}} H^{\operatorname{codim} Y}(\pi: \Gamma) .
$$

Hence $\operatorname{pr}_{1}(\mathcal{M}(Y))=\frac{\operatorname{vol}(Y)}{\operatorname{vol}(X)} \Psi\left(\mathcal{M}\left(Y_{U}\right)\right)$.

\section{$\S 4$. An explicit vanishing theorem for modular symbols}

Any irreducible, infinitesimally unitarizable $(\mathfrak{g}, K)$-module with non-zero $(\mathfrak{g}, K)$ cohomology groups is represented as Vogan-Zuckerman's derived functor module $A_{\mathfrak{q}}([26])$. Therefore, in order to apply Theorem 2.8 for actual computation of modular symbols, it is important to tell an explicit condition such that $\left.\overline{A_{\mathfrak{q}}}\right|_{K^{\prime}}$ is $K^{\prime}$-admissible. Such a criterion was studied in [6] and [7]. Thus we can reformulate the vanishing theorem for modular symbols defined by a symmetric pair with an explicit condition by means of the root system. These are the main ingredients of this section (see Theorem 4.3).

Let $G$ be a real reductive linear Lie group. We recall the notation in $\S 2$. In particular, $\mathfrak{g}_{0}=\mathfrak{k}_{0}+\mathfrak{p}_{0}$ is a Cartan decomposition and $\mathfrak{g}=\mathfrak{k}+\mathfrak{p}$ denotes the complexifications. Take a Cartan subalgebra $\mathfrak{t}_{0}$ of $\mathfrak{k}_{0}$. Let

$$
\mathfrak{g}(\mathfrak{t} ; \alpha):=\{X \in \mathfrak{g}:[H, X]=\alpha(H) X \text { for } H \in \mathfrak{t}\} .
$$

Given an element $\lambda \in \sqrt{-1} t_{0}^{*}$. Let

$$
\begin{aligned}
L & \equiv L(\lambda):=\left\{g \in G: \operatorname{Ad}^{*}(g) \lambda=\lambda\right\}, \\
\mathfrak{l} & \equiv \mathfrak{l}(\lambda):=\operatorname{Lie}(L(\lambda)) \underset{\mathbb{R}}{\otimes} \mathbb{C}=\sum_{\langle\lambda, \alpha\rangle=0} \mathfrak{g}(\mathfrak{t} ; \alpha), \\
\mathfrak{u} & \equiv \mathfrak{u}(\lambda):=\sum_{\langle\lambda, \alpha\rangle>0} \mathfrak{g}(\mathfrak{t} ; \alpha), \\
\overline{\mathfrak{u}} & \equiv \overline{\mathfrak{u}}(\lambda):=\sum_{\langle\lambda, \alpha\rangle<0} \mathfrak{g}(\mathfrak{t} ; \alpha), \\
\mathfrak{q} & \equiv \mathfrak{q}(\lambda):=\mathfrak{l}(\lambda)+\mathfrak{u}(\lambda) .
\end{aligned}
$$

The subalgebra $\mathfrak{q} \equiv \mathfrak{q}(\lambda)$ is said to be a $\theta$-stable subalgebra of $\mathfrak{g}([23])$. The homogeneous manifold $G / L(\lambda)$ carries a $G$-invariant complex structure with the holomorphic tangent bundle being $G \times_{L(\lambda)} \overline{\mathfrak{u}}(\lambda)$. We define $A_{\mathfrak{q}}$ to be the underlying $(\mathfrak{g}, K)$-module of the $S:=\operatorname{dim}_{\mathbb{C}} K / L \cap K$-th Dolbeault cohomology group $H \frac{S}{\partial}(G / L(\lambda), \Omega)$ with the coefficient in the canonical line bundle $\Omega \equiv \Omega_{G / L(\lambda)}$ 
([20], [28]). Then $A_{\mathfrak{q}}$ is an irreducible Harish-Chandra module which is unitarizable ([24], [27]). The corresponding unitary representation is denoted by $\overline{A_{\mathfrak{q}}} \in \widehat{G}_{0}$ $(\subset \widehat{G})$. Then it is a result due to Vogan-Zuckerman $([26])$ that

$$
\widehat{G}_{\mathrm{VZ}}:=\left\{A_{\mathfrak{q}}: \mathfrak{q} \text { is a } \theta \text {-stable parabolic subalgebra of } \mathfrak{g}\right\}\left(\subset \widehat{G}_{0} \subset \widehat{G}\right)
$$

is the totality of irreducible unitary representations of $G$ whose underlying $(\mathfrak{g}, K)$ modules have non-zero $(\mathfrak{g}, K)$-cohomology groups. We note that if $\mathfrak{q}$ is a Borel subalgebra and if $\operatorname{rank} G=\operatorname{rank} K$ then $\overline{A_{\mathfrak{q}}}$ is a discrete series representation of $G$.

Suppose $\widetilde{\mathfrak{q}}=\widetilde{\mathfrak{l}}+\widetilde{\mathfrak{u}}$ and $\mathfrak{q}=\mathfrak{l}+\mathfrak{u}$ are $\theta$-stable parabolic subalgebras such that $\widetilde{\mathfrak{l}} \supset \mathfrak{l}$ and $\widetilde{\mathfrak{u}} \subset \mathfrak{u}$. If $\widetilde{L} / L$ is compact, then there is a compact normal subgroup $L^{\prime}$ of $\widetilde{L}$ such that $\widetilde{L}=L^{\prime} L$. Then we have $A_{\widetilde{q}} \simeq A_{\mathfrak{q}}$ because the spectral sequence of induction by stages ([23], Proposition 6.3.6) collapses thanks to the Borel-WeilBott theorem for the compact group $L^{\prime}$. So, we consider the following condition on a $\theta$-stable parabolic subalgebra $\mathfrak{q}$ :

There exists no $\theta$-stable parabolic subalgebra $\widetilde{\mathfrak{q}}=\widetilde{\mathfrak{l}}+\widetilde{\mathfrak{u}}$ of $\mathfrak{g}$ that satisfies both (4.1.1) and (4.1.2):

$$
\begin{aligned}
& \widetilde{\mathfrak{l}} \supsetneqq \mathfrak{l}, \quad \widetilde{\mathfrak{u}} \varsubsetneqq \mathfrak{u}, \\
& \widetilde{L} / L \text { is compact. }
\end{aligned}
$$

Then, the set of $\theta$-stable parabolic subalgebras $\mathfrak{q}$ satisfying (4.1) is sufficient to describe $\widehat{G}_{\mathrm{VZ}}$. That is,

$$
\widehat{G}_{\mathrm{VZ}}=\left\{A_{\mathfrak{q}}: \mathfrak{q} \text { is a } \theta \text {-stable parabolic subalgebra of } \mathfrak{g} \text { satisfying }(4.1)\right\} .
$$

Next, we suppose that $\sigma$ is an involutive automorphism of $G$. If $G^{\prime}$ is an open subgroup of $G^{\sigma}:=\{g \in G: \sigma g=g\}$, then the pair $\left(G, G^{\prime}\right)$ is said to be a reductive symmetric pair (or a semisimple symmetric pair if $G$ is semisimple). We fix a Cartan involution $\theta$ of $G$ such that $\sigma \theta=\theta \sigma$. Then $\theta G^{\prime}=G^{\prime}, K^{\prime}:=K \cap G^{\prime}$ is a maximal compact subgroup of $G^{\prime}$ and the pair $\left(K, K^{\prime}\right)$ forms a compact symmetric pair. We write $\mathfrak{k}_{0 \pm}:=\left\{X \in \mathfrak{k}_{0}: \sigma(X)= \pm X\right\}$. We take a $\sigma$-stable Cartan subalgebra $\mathfrak{t}_{0}$ of $\mathfrak{k}_{0}$ so that $\mathfrak{t}_{0-}:=\mathfrak{t}_{0} \cap \mathfrak{k}_{0-}$ is a maximal abelian subspace in $\mathfrak{k}_{0-}$. Let $\Delta(\mathfrak{k}, \mathfrak{t})$ be the root system and $\Sigma\left(\mathfrak{k}, \mathfrak{t}_{-}\right)$the restricted root system of $\mathfrak{k}$ with respect to $\mathfrak{t}$ and $\mathfrak{t}_{-}$, respectively. We fix a positive system $\Sigma^{+}\left(\mathfrak{k}, \mathfrak{t}_{-}\right)$ and take a positive system $\Delta^{+}(\mathfrak{k}, \mathfrak{t})$ which is compatible with $\Sigma^{+}\left(\mathfrak{k}, \mathfrak{t}_{-}\right)$. We shall regard as $\sqrt{-1}\left(\mathfrak{t}_{0-}\right)^{*} \subset \sqrt{-1} \mathfrak{t}_{0}^{*}$ according to the direct sum decomposition $\mathfrak{t}_{0}=$ $\mathfrak{t}_{0-}+\left(\mathfrak{t}_{0} \cap \mathfrak{k}_{0+}\right)$.

Suppose $\mathfrak{q}=\mathfrak{q}(\lambda)$ is a $\theta$-stable parabolic subalgebra of $\mathfrak{g}$ defined by $\lambda \in \sqrt{-1} \mathfrak{t}_{0}^{*}$ as before. After conjugation by an element of $K$, we may and do assume that 
$\lambda \in \sqrt{-1} \mathfrak{t}_{0}^{*}$ is dominant with respect to $\Delta^{+}(\mathfrak{k}, \mathfrak{t})$. Then a closed cone $\sqrt{-1} \mathfrak{t}_{0}^{*}$ is defined by

$$
\mathbb{R}_{+}\langle\mathfrak{u} \cap \mathfrak{p}\rangle:=\left\{\sum_{\beta \in \Delta(\mathfrak{u} \cap \mathfrak{p} ; \mathfrak{t})} n_{\beta} \beta: n_{\beta} \geq 0\right\} \subset \sqrt{-1} \mathfrak{t}_{0}^{*} .
$$

Fact 4.2. Retain the notation as above. Then the following three conditions on $\left(\mathfrak{q}, G, G^{\prime}\right)$ are equivalent:

1) $\mathbb{R}_{+}\langle\mathfrak{u} \cap \mathfrak{p}\rangle \cap \sqrt{-1}\left(\mathfrak{t}_{0_{-}}\right)^{*}=\{0\}$.

2) $\left.\overline{A_{\mathfrak{q}}}\right|_{K^{\prime}}$ is $K^{\prime}$-admissible.

3) $A_{\mathfrak{q}}$ is discretely decomposable as a $\left(\mathfrak{g}^{\prime}, K^{\prime}\right)$-module.

Proof. See [6], Theorem 3.2 for $(1) \Longrightarrow(2)$; and [8], Theorem 4.2 for other implications.

Now, we have

Theorem 4.3. Let $\left(G, G^{\prime}\right)$ be a reductive symmetric pair, and $\Gamma$ a torsion-free, co-compact discrete subgroup of $G$ such that $\Gamma^{\prime}:=\Gamma \cap G^{\prime}$ is co-compact in $G^{\prime}$. Suppose that $\mathfrak{q}=\mathfrak{l}+\mathfrak{u}$ is a $\theta$-stable parabolic subalgebra of $\mathfrak{g}$. Assume the following three conditions are satisfied:

i) $\mathfrak{l} \not \supset \mathfrak{p}$,

ii) $\mathbb{R}_{+}\langle\mathfrak{u} \cap \mathfrak{p}\rangle \cap \sqrt{-1}\left(\mathfrak{t}_{0_{-}}\right)^{*}=\{0\}$,

iii) $G^{\prime}$ is semisimple without compact factors.

Then we have

$$
\left\langle H^{\mathrm{d}\left(G^{\prime}\right)}\left(\overline{A_{\mathfrak{q}}}: \Gamma\right), \iota_{*}\left(\left[\Gamma^{\prime} \backslash G^{\prime} / K^{\prime}\right]\right)\right\rangle=0 .
$$

Proof. The assumption (i) implies that $\overline{A_{\mathfrak{q}}}$ is infinite dimensional. By Fact 4.2 , the restriction ${\overline{A_{\mathfrak{q} \mid}}}_{K^{\prime}}$ is $K^{\prime}$-admissible because of the assumption (ii). Now Theorem 4.3 is an immediate consequence of Theorem 2.8.

Remark 4.4. If $\mathfrak{q}$ satisfies (4.1) then the assumption (i) in Theorem 4.3 is equivalent to the condition that $\mathfrak{l} \neq \mathfrak{g}$.

Remark 4.5. We can generalize Theorem 4.3 to the non-symmetric case by using [7], Theorem 2.9.

\section{$\S 5$. Modular symbols on Hermitian symmetric spaces of type IV}

In this section, we apply the results in $\S 2, \S 3$ and $\S 4$ to the symmetric pair

$$
\left(G, G^{\prime}\right)=\left(S O_{0}(2 n, 2), S O_{0}(2 n, 1)\right) .
$$


Here, $S O_{0}(p, q)$ denotes the identity component of the orthogonal group $O(p, q)$ of signature $(p, q)$. We shall study the middle Hodge component of the modular symbol defined by $G^{\prime}$. The key step is the following:

Lemma 5.1. If the $\pi_{K}$ is a unitarizable irreducible $(\mathfrak{g}, K)$-module such that

$$
H^{n, n}\left(\mathfrak{g}, K ; \pi_{K}\right) \neq 0,
$$

then $\left.\pi_{K}\right|_{K^{\prime}}$ is $K^{\prime}$-admissible. In particular, $\pi_{K}$ is discretely decomposable as a $\left(\mathfrak{g}^{\prime}, K^{\prime}\right)$-module.

This lemma will be proved after Lemma 5.3, 5.4 and 5.5. First we fix notation. A maximal compact subgroup $K$ of $G$ is isomorphic to $S O(2 n) \times S O(2)$. Take a Cartan subalgebra $\mathfrak{t}_{0}$ of $\mathfrak{k}_{0}=$ Lie $K$, which is also a Cartan subalgebra $\mathfrak{t}_{0}$ of $\mathfrak{g}_{0}$ because of $\operatorname{rank} K=\operatorname{rank} G=n+1$. We take a basis $\left\{f_{1}, \cdots, f_{n+1}\right\}$ of $\sqrt{-1} \mathrm{t}_{0}^{*}$ so that the root systems of $\mathfrak{k}$ and $\mathfrak{g}$ are represented as

$$
\begin{aligned}
\Delta(\mathfrak{k}, \mathfrak{t}) & =\left\{ \pm\left(f_{i} \pm f_{j}\right): 1 \leq i<j \leq n\right\} \subset \sqrt{-1} \mathfrak{t}_{0}^{*}, \\
\Delta(\mathfrak{g}, \mathfrak{t}) & =\left\{ \pm\left(f_{i} \pm f_{j}\right): 1 \leq i<j \leq n+1\right\} \subset \sqrt{-1} \mathfrak{t}_{0}^{*} .
\end{aligned}
$$

Let $\mathfrak{g}=\mathfrak{k}+\mathfrak{p}=\mathfrak{k} \oplus \mathfrak{p}_{+} \oplus \mathfrak{p}_{-}$be the irreducible decomposition as $\operatorname{Ad}(K)$-modules where the subspaces $\mathfrak{p}_{ \pm}$are defined by the weights with respect to $\mathfrak{t}$ as follows:

$$
\begin{aligned}
\Delta\left(\mathfrak{p}_{+}, \mathfrak{t}\right) & =\left\{ \pm f_{i}+f_{n+1}: 1 \leq i \leq n\right\}, \\
\Delta\left(\mathfrak{p}_{-}, \mathfrak{t}\right) & =\left\{ \pm f_{i}-f_{n+1}: 1 \leq i \leq n\right\} .
\end{aligned}
$$

Here we note that $\mathfrak{p}_{ \pm}$are $\operatorname{Ad}(K)$-stable, and consequently ad( $\left.\mathfrak{t}\right)$-stable.

We first define some special $\theta$-stable parabolic subalgebras of $\mathfrak{g}$, denoted by $\mathfrak{q}_{0}, \cdots, \mathfrak{q}_{n-1}, \mathfrak{q}_{n}^{ \pm}$, which contribute $(\mathfrak{g}, K)$-cohomology groups of degree $(n, n)$ (see Lemma 5.3 and Lemma 5.4). We fix a positive system of $\Delta(\mathfrak{k}, \mathfrak{t})$ once and for all as follows:

$$
\Delta^{+}(\mathfrak{k}, \mathfrak{t}):=\left\{f_{i} \pm f_{j}: 1 \leq i<j \leq n\right\} .
$$

For $k=0,1, \cdots, n-1$, we take $\lambda=\sum_{i=1}^{n+1} \lambda_{i} f_{i} \in \sqrt{-1} \mathrm{t}_{0}^{*}$ so that

$$
\lambda_{1}>\cdots>\lambda_{k}>\lambda_{k+1}=\cdots=\lambda_{n}=\lambda_{n+1}=0 .
$$

According to $\S 4$, we define a $\theta$-stable parabolic subalgebra of $\mathfrak{g}$ by

$$
\mathfrak{q}_{k}:=\mathfrak{q}(\lambda) .
$$

This definition is independent of the choice of $\lambda$ as far as $\lambda$ satisfies (5.2.1). Note that $\mathfrak{q}_{0}=\mathfrak{g}$. 
Similarly, we take $\lambda=\sum_{i=1}^{n+1} \lambda_{i} f_{i} \in \sqrt{-1} \mathrm{t}_{0}^{*}$ so that

$$
\lambda_{1}>\cdots>\lambda_{n-1}>\left|\lambda_{n}\right|>\lambda_{n+1}=0
$$

and define a $\theta$-stable subalgebra $\mathfrak{q}(\lambda)$. This definition depends only on the signature of $\lambda_{n}$. So, we write

$$
\mathfrak{q}_{n}^{\epsilon}:=\mathfrak{q}(\lambda), \quad \epsilon:=\operatorname{sgn} \lambda_{n} \in\{ \pm\}
$$

Then Levi subgroups corresponding to $\mathfrak{q}_{k}, \mathfrak{q}_{n}^{ \pm}$are given respectively as follows:

$$
\begin{aligned}
L_{k} & \simeq \mathbb{T}^{k} \times S O_{0}(2 n-2 k, 2) \quad(0 \leq k \leq n-1), \\
L_{n}^{ \pm} & \simeq \mathbb{T}^{n} .
\end{aligned}
$$

The unitary representations $\overline{A_{\mathfrak{q}_{n}^{ \pm}}}$are discrete series representations for $G$, while $\overline{A_{\mathfrak{q}_{k}}}$ are non-tempered representations of $G$. We recall that if $G / K$ is a Hermitian symmetric space, then the Hodge component of $(\mathfrak{g}, K)$-cohomology groups is given by

$$
H^{p, q}\left(\mathfrak{g}, K ; A_{\mathfrak{q}}\right) \simeq H^{p+R_{+}, q+R_{-}}(\mathfrak{l}, L \cap K ; \mathbb{C})
$$

where $R_{ \pm}:=\operatorname{dim}\left(\mathfrak{u} \cap \mathfrak{p}_{ \pm}\right.$) (see [3]). For the reader's convenience, we present the computation of $(\mathfrak{g}, K)$-cohomology groups of $A_{\mathfrak{q}_{k}}(0 \leq k \leq n-1)$ and $A_{\mathfrak{q}_{n}^{ \pm}}$.

Lemma 5.3. (see [12], [26]). Let $0 \leq k \leq n-1$.

$$
\begin{aligned}
& H^{i, j}\left(\mathfrak{g}, K ; A_{\mathfrak{q}_{k}}\right)= \begin{cases}\mathbb{C}+\mathbb{C} & \text { if } i=j=n, \\
\mathbb{C} & \text { if } k \leq i=j \leq 2 n-k, i \neq n, \\
0 & \text { otherwise, }\end{cases} \\
& H^{i, j}\left(\mathfrak{g}, K ; A_{\mathfrak{q}_{n}^{ \pm}}\right) \simeq \begin{cases}\mathbb{C} & \text { if } i=j=n, \\
0 & \text { otherwise. }\end{cases}
\end{aligned}
$$

Thus, $A_{\mathfrak{q}_{k}}(0 \leq k \leq n-1)$ and $A_{\mathfrak{q}_{n}^{ \pm}}$contribute to the $(\mathfrak{g}, K)$-cohomology groups of degree $(n, n)$. Conversely, no other irreducible infinitesimally unitarizable $(\mathfrak{g}, K)$-modules contribute $(\mathfrak{g}, K)$-cohomology groups of degree $(n, n)$. That is, we have the following:

Lemma 5.4. Let $G=S O_{0}(2 n, 2)$, and $\pi_{K}$ an irreducible unitarizable $(\mathfrak{g}, K)$ module such that

$$
H^{n, n}\left(\mathfrak{g}, K ; \pi_{K}\right) \neq 0 .
$$

Then $\pi_{K} \simeq A_{\mathfrak{q}}$ as $(\mathfrak{g}, K)$-modules, where $\mathfrak{q}$ is one of $\mathfrak{q}_{0}, \mathfrak{q}_{1}, \cdots, \mathfrak{q}_{n-1}, \mathfrak{q}_{n}^{+}$or $\mathfrak{q}_{n}^{-}$. 
Proof. By a theorem of Vogan-Zuckerman [26], $\pi_{K}$ is isomorphic to $A_{\mathfrak{q}}$ where $\mathfrak{q}=\mathfrak{l}+\mathfrak{u}$ some $\theta$-stable parabolic subalgebra of $\mathfrak{g}=\operatorname{Lie}\left(S O_{0}(2 n, 2)\right) \otimes_{\mathbb{R}} \mathbb{C}$. We may assume that $\mathfrak{q}$ satisfies (4.1). The condition (5.4.1) implies that

$$
\operatorname{dim}\left(\mathfrak{u} \cap \mathfrak{p}_{+}\right)=\operatorname{dim}\left(\mathfrak{u} \cap \mathfrak{p}_{-}\right) .
$$

We shall prove that $\mathfrak{q}$ is conjugate to one of $\mathfrak{q}_{0}, \mathfrak{q}_{1}, \cdots, \mathfrak{q}_{n-1}, \mathfrak{q}_{n}^{+}$or $\mathfrak{q}_{n}^{-}$by the adjoint action of $K$ if $\mathfrak{q}$ satisfies (4.1) and (5.4.1'). After a conjugation by an element of $K$, we may assume that $\mathfrak{q} \equiv \mathfrak{q}(\lambda)=\mathfrak{l}+\mathfrak{u} \equiv \mathfrak{l}(\lambda)+\mathfrak{u}(\lambda)$ is defined by $\lambda$ that is dominant with respect to $\Delta^{+}(\mathfrak{k}, \mathfrak{t})$. That is, if we write

$$
\lambda=\sum_{i=1}^{n+1} \lambda_{i} f_{i} \in \sqrt{-1} \mathfrak{t}_{0}^{*}
$$

then we have $\lambda_{1} \geq \cdots \geq \lambda_{n-1} \geq\left|\lambda_{n}\right|$, since the root system $\Delta(\mathfrak{k}, \mathfrak{t})$ is of type $D_{n}$. Then we have

$$
\begin{aligned}
\Delta\left(\mathfrak{u}(\lambda) \cap \mathfrak{p}_{+}, \mathfrak{t}\right)= & \left\{f_{i}+f_{n+1}: 1 \leq i \leq n, \lambda_{i}+\lambda_{n+1}>0\right\} \\
& \cup\left\{-f_{i}+f_{n+1}: 1 \leq i \leq n,-\lambda_{i}+\lambda_{n+1}>0\right\}, \\
\Delta\left(\mathfrak{u}(\lambda) \cap \mathfrak{p}_{-}, \mathfrak{t}\right)= & \left\{f_{i}-f_{n+1}: 1 \leq i \leq n, \lambda_{i}-\lambda_{n+1}>0\right\} \\
& \cup\left\{-f_{i}-f_{n+1}: 1 \leq i \leq n,-\lambda_{i}-\lambda_{n+1}>0\right\}
\end{aligned}
$$

Therefore, the assumption $\operatorname{dim}\left(\mathfrak{u}(\lambda) \cap \mathfrak{p}_{+}\right)=\operatorname{dim}\left(\mathfrak{u}(\lambda) \cap \mathfrak{p}_{-}\right)$amounts to

$$
\begin{aligned}
& \#\left\{i: 1 \leq i \leq n, \lambda_{i}+\lambda_{n+1}>0\right\}+\#\left\{i: 1 \leq i \leq n,-\lambda_{i}+\lambda_{n+1}>0\right\} \\
& =\#\left\{i: 1 \leq i \leq n, \lambda_{i}-\lambda_{n+1}>0\right\}+\#\left\{i: 1 \leq i \leq n,-\lambda_{i}-\lambda_{n+1}>0\right\} .
\end{aligned}
$$

We set

$$
\begin{aligned}
k & :=\#\left\{i: 1 \leq i \leq n,\left|\lambda_{i}\right|>\left|\lambda_{n+1}\right|\right\} \in\{0,1, \cdots, n\}, \\
m & :=\#\left\{i: 1 \leq i \leq n,\left|\lambda_{i}\right|=\left|\lambda_{n+1}\right|\right\} \in\{0,1, \cdots, n\} .
\end{aligned}
$$

Obviously $k+m \leq n$. Without loss of generality, we may assume $\lambda_{n+1} \geq 0$ hereafter. Then the definition of $k$ and $m$ leads to

$$
\left\{\begin{array}{lc}
\lambda_{1} \geq \cdots \geq \lambda_{k}>\lambda_{k+1}=\cdots=\lambda_{k+m}=\lambda_{n+1} & \\
\quad>\lambda_{k+m+1} \geq \cdots \geq \lambda_{n-1} \geq\left|\lambda_{n}\right| & (k+m<n), \\
\lambda_{1} \geq \cdots \geq \lambda_{k}>\lambda_{k+1}=\cdots=\lambda_{n-1}=\left|\lambda_{n}\right|=\lambda_{n+1} & (k+m=n, k \neq n), \\
\lambda_{1} \geq \cdots \geq \lambda_{n-1} \geq\left|\lambda_{n}\right|>\lambda_{n+1} & (k+m=n, k=n) .
\end{array}\right.
$$

Then it is an elementary computation to see

$$
\text { the left side of }(5.4 .2)= \begin{cases}n+(n-k-m) & \text { if } k+m<n, \\ n & \text { if } k+m=n, \lambda_{n+1}>0 \\ k & \text { if } k+m=n, \lambda_{n+1}=0\end{cases}
$$


the right side of $(5.4 .2)=k$.

In view of $k+m \leq n$, the equation (5.4.2) holds if and only if either (5.4.3) or (5.4.4) is satisfied:

$$
\begin{aligned}
& k+m=n, \lambda_{n+1}>0, k=n, \\
& k+m=n, \lambda_{n+1}=0 .
\end{aligned}
$$

Then (5.4.3) and (5.4.4) imply

$$
\begin{aligned}
& \lambda_{1} \geq \cdots \geq \lambda_{n-1} \geq\left|\lambda_{n}\right| \geq \lambda_{n+1}>0, \\
& \lambda_{1} \geq \cdots \geq \lambda_{k}>\lambda_{k+1}=\cdots=\lambda_{n}=\lambda_{n+1}=0,
\end{aligned}
$$

respectively. Let us compare $\mathfrak{q} \equiv \mathfrak{q}(\lambda)$ with $\mathfrak{q}_{0}, \mathfrak{q}_{1}, \cdots, \mathfrak{q}_{n-1}, \mathfrak{q}_{n}^{ \pm}$. If $\lambda$ satisfies (5.4.3'), then $\mathfrak{q} \supset \mathfrak{q}_{n}^{+}$and $L / L_{n}^{+}$is compact. If $\lambda$ satisfies $\left(5.4 .4^{\prime}\right)$, then $\mathfrak{q} \supset \mathfrak{q}_{k}$ and $L / L_{k}$ is compact. Because $\mathfrak{q}$ satisfies $(4.1), \lambda=\left(\lambda_{1}, \cdots, \lambda_{n+1}\right)$ must satisfy

$$
\begin{aligned}
& \lambda_{1}>\cdots>\lambda_{n-1}>\left|\lambda_{n}\right|>\lambda_{n+1}>0, \\
& \lambda_{1}>\cdots>\lambda_{k}>\lambda_{k+1}=\cdots=\lambda_{n}=\lambda_{n+1}=0,
\end{aligned}
$$

respectively, which means $\mathfrak{q}=\mathfrak{q}_{n}^{+}$or $\mathfrak{q}=\mathfrak{q}_{k}(1 \leq k \leq n-1)$. This is what we wanted to prove. The case $\lambda_{n+1} \leq 0$ is similar.

Lemma 5.5. Suppose that a $\theta$-stable parabolic subalgebra $\mathfrak{q}=\mathfrak{l}+\mathfrak{u}$ of $\mathfrak{g}$ is one of $\mathfrak{q}_{0}, \mathfrak{q}_{1}, \cdots, \mathfrak{q}_{n-1}, \mathfrak{q}_{n}^{ \pm}$. Then we have

$$
\mathbb{R}_{+}\langle\mathfrak{u} \cap \mathfrak{p}\rangle \cap \mathbb{R} f_{n+1}=\{0\} .
$$

Proof. The set of weights for $\mathfrak{u} \cap \mathfrak{p}$ with respect to a Cartan subalgebra $\mathfrak{t}$ of $\mathfrak{k}$ is given by

$$
\Delta(\mathfrak{u} \cap \mathfrak{p}, \mathfrak{t})= \begin{cases}\left\{f_{i} \pm f_{n+1}: 1 \leq i \leq k\right\} & \text { if } \mathfrak{q}=\mathfrak{q}_{k} \\ \left\{f_{i} \pm f_{n+1}: 1 \leq i \leq n-1\right\} \cup\left\{\epsilon f_{n} \pm f_{n+1}\right\} & \text { if } \mathfrak{q}=\mathfrak{q}_{n}^{\epsilon}\end{cases}
$$

where $0 \leq k \leq n-1$ and $\epsilon= \pm$. In either case, we have

$$
\begin{aligned}
& \mathbb{R}_{+}\langle\mathfrak{u} \cap \mathfrak{p}\rangle=\left\{\sum_{\alpha \in \Delta(\mathfrak{u} \cap \mathfrak{p}, \mathfrak{t})} a_{\alpha} \alpha: a_{\alpha} \geq 0\right\} \\
& \subset\left\{\sum_{i=1}^{n-1}\left(a_{i}+b_{i}\right) f_{i}+\epsilon\left(a_{n}+b_{n}\right) f_{n}+\left(\sum_{i=1}^{n}\left(a_{i}-b_{i}\right)\right) f_{n+1}: a_{i}, b_{i} \geq 0,1 \leq i \leq n\right\} .
\end{aligned}
$$


Therefore

$$
\begin{aligned}
& \mathbb{R}_{+}\langle\mathfrak{u} \cap \mathfrak{p}\rangle \cap \mathbb{R} f_{n+1} \\
& \subset\left\{\sum_{i=1}^{n-1}\left(a_{i}+b_{i}\right) f_{i}+\epsilon\left(a_{n}+b_{n}\right) f_{n}+\left(\sum_{i=1}^{n}\left(a_{i}-b_{i}\right)\right) f_{n+1}: a_{i}, b_{i} \geq 0\right\} \cap \mathbb{R} f_{n+1} .
\end{aligned}
$$

If $\sum_{i=1}^{n-1}\left(a_{i}+b_{i}\right) f_{i}+\epsilon\left(a_{n}+b_{n}\right) f_{n}+\left(\sum_{i=1}^{n}\left(a_{i}-b_{i}\right)\right) f_{n+1} \in \mathbb{R} f_{n+1}$, then $a_{i}+b_{i}=0$ $(1 \leq i \leq n-1)$ and $\epsilon\left(a_{n}+b_{n}\right)=0$. This holds if and only if $a_{i}=b_{i}=0$ for all $i$ $(1 \leq i \leq n)$ because $a_{i}, b_{i} \geq 0$. Hence $\mathbb{R}_{+}\langle\mathfrak{u} \cap \mathfrak{p}\rangle \cap \mathbb{R} f_{n+1}=\{0\}$.

Now, we are ready to prove Lemma 5.1:

Proof of Lemma 5.1. Suppose that $\pi_{K}$ is an irreducible $(\mathfrak{g}, K)$-module satisfying $H^{n, n}\left(\mathfrak{g}, K ; \pi_{K}\right) \neq 0$. It follows from Lemma 5.4 that $\pi_{K} \simeq A_{\mathfrak{q}}$ as $(\mathfrak{g}, K)$-modules, where $\mathfrak{q}$ is one of $\mathfrak{q}_{0}, \cdots, \mathfrak{q}_{n-1}$ or $\mathfrak{q}_{n}^{ \pm}$.

On the other hand, in view of $\left(K, K^{\prime}\right)=(S O(2 n) \times S O(2), S O(2 n))$, we have

$$
\sqrt{-1}\left(\mathfrak{t}_{0_{-}}\right)^{*}=\mathbb{R} f_{n+1} \text {. }
$$

Here, we recall that $\left\{f_{1}, \cdots, f_{n+1}\right\}$ is a basis of $\sqrt{-1} \mathrm{t}_{0}^{*}$ (see $\S 4$ for the definition of $\left.\mathfrak{t}_{0_{-}}\right)$. Hence

$$
\mathbb{R}_{+}\langle\mathfrak{u} \cap \mathfrak{p}\rangle \cap \sqrt{-1}\left(\mathfrak{t}_{0_{-}}\right)^{*}=\{0\}
$$

by Lemma 5.5. Therefore, it follows from Fact 4.2 that $\left.A_{\mathfrak{q}}\right|_{K^{\prime}}$ is $K^{\prime}$-admissible.

Theorem 5.6. Let $\Gamma$ be a torsion-free discrete subgroup of $G=S O_{0}(2 n, 2), G^{\prime}:=$ $S O_{0}(2 n, 1) \subset G$ and $\Gamma^{\prime}:=\Gamma \cap G^{\prime}$. Assume that $G / \Gamma$ and $G^{\prime} / \Gamma^{\prime}$ are compact. Let $\iota: Y=\Gamma^{\prime} \backslash G^{\prime} / K^{\prime} \rightarrow X=\Gamma \backslash G / K$ be the natural map as before. Let $\pi(\neq \mathbf{1})$ be an irreducible unitary representation of $G$. We write

$$
\operatorname{pr}_{1}: H_{\text {de } \operatorname{Rham}}^{*}(X ; \mathbb{C}) \rightarrow H^{*}(\mathbf{1}: \Gamma)=H^{*}(\mathfrak{g}, K ; \mathbb{C})
$$

for the projection onto 1-component in the Matsushima-Murakami formula (see Lemma 2.4) and

$$
\mathcal{M}(Y)=\sum_{p+q=2 n} \mathcal{M}^{p, q}(Y) \in \bigoplus_{p+q=2 n} H^{p, q}(X ; \mathbb{C})
$$

for the Hodge decomposition of the Poincaré dual of the modular symbol $\iota_{*}([Y]) \in$ $H_{2 n}(X ; \mathbb{C})$. Then we have

$$
\mathcal{M}^{n, n}(Y)=\operatorname{pr}_{\mathbf{1}}(\mathcal{M}(Y))
$$


Proof. By Corollary 2.10 for the subspace $H^{n, n}(X ; \mathbb{C})$ of $H_{\text {de }}^{2 n}$ Rham $(X ; \mathbb{C})$, it suffices to show

$$
\left\langle H^{2 n}(\pi: \Gamma), \iota_{*}\left(\left[\Gamma^{\prime} \backslash G^{\prime} / K^{\prime}\right]\right)\right\rangle=0
$$

for any $\pi \in \widehat{G}$ such that $\pi \neq \mathbf{1}$ and $H^{n, n}\left(\mathfrak{g}, K ; \pi_{K}\right) \neq 0$. Because $\pi$ is not the trivial representation, we have $\mathfrak{q} \neq \mathfrak{g}$ and so $\mathfrak{l} \not \supset \mathfrak{p}$. It follows from Lemma 5.1 and Theorem 4.3 that

$$
\left\langle H^{2 n}(\pi: \Gamma), \iota_{*}\left(\left[\Gamma^{\prime} \backslash G^{\prime} / K^{\prime}\right]\right)\right\rangle=0,
$$

which we wanted to prove.

Now Theorem in Introduction follows from Theorem 3.1 and Theorem 5.6.

\section{$\S$ 6. Concluding remarks}

Remark 6.1. Before finishing this paper, let us note some heuristic argument on the relation between our result and the Rankin convolution method which is used to obtain an integral expression of certain automorphic $L$-functions and to represent the special values of such $L$ in terms of some trajectory integrals. The typical situation is the following. Let $\Phi$ be an automorphic form on the adelization $G_{\mathbb{A}}$ of a reductive algebraic group $G$ defined over a global field. Let $H$ be an algebraic subgroup of $G$. Then we consider some Eisenstein series $E(s, h)$ on $\mathbb{C} \times H_{\mathbb{A}}$ and another cusp form $\psi$ on $H_{\mathbb{A}}$. Then we consider the integral

$$
\int_{H_{k} \backslash H_{\mathbb{A}}} \Phi(h) \psi(h) E(s, h) d h .
$$

The basic assumption behind, but which rarely referred explicitly, is that the tensor product of automorphic representations

$$
\left(\left.\Pi_{\Phi}\right|_{H_{\AA}}\right) \otimes \pi_{\psi}
$$

has a continuous spectrum. Here we denote by $\Pi_{\Phi}$ and $\pi_{\psi}$ the automorphic representations of $G_{\mathbb{A}}$ and $H_{\mathbb{A}}$, respectively, generated by $\Phi$ and $\psi$. Then $G_{\mathbb{R}}$ is a real reductive linear group, corresponding to the notation " $G$ " in this paper. Suppose that $\psi$ is the constant. Then the very assumption on the existence of continuous spectrum for the restriction $\left.\Pi_{\Phi}\right|_{H_{\mathbb{A}}}$ fails if the real component $\Pi_{\mathbb{R}}$ of $\Pi_{\Phi}$ satisfies the criterion Fact 4.2, because of the discrete decomposability of the restriction $\left.\Pi_{\mathbb{R}}\right|_{H_{\mathbb{R}}}$. So the Rankin convolution gives just zero in this case!

Remark 6.2. If $G / K$ is Hermitian, then the arithmetic quotient $V=\Gamma \backslash G / K$ is an algebraic variety by a classical result of Baily. When $\Gamma$ is not co-compact in $G$, modular embeddings $H \subset G$ do not give cycles on $V$, and they are just chains in 
general. Consider the Baily-Borel-Satake compactification $V^{*}$ of $V$. Then we can form the closure of modular symbols in $V^{*}$ and obtain closed real analytic subsets in $V^{*}$. One can investigate the perversity of these compactified modular symbols (at least) in some special cases, in the sense of Goretzky-MacPherson. When $G=S O_{0}(m, 2)$ and $H=S O_{0}(m, 1)$, they never have the middle perversity. We refer to a related result in the case $m=3$, by Nygaard [15], Theorem 2.1, which was brought to our attention by Dr. Miyazaki. It is an easy exercise to check that the closure of modular symbols has "one-step worse" perversity than the middle perversity. This fact is interesting because it may give an account of the "failure of the Petersson conjecture" for the lifted modular forms and the mixed cohomology associated to such modular forms (cf. [17], Conjecture 5.4).

Remark 6.3. Suppose we are in the setting of $\S 5$. In contrast to Lemma 5.1, not all unitarizable irreducible $(\mathfrak{g}, K)$-modules $\pi_{K}$ that contribute to the $(p, q)$-type Hodge component $H^{p, q}\left(\mathfrak{g}, K ; \pi_{K}\right)$ are discretely decomposable as $\left(\mathfrak{g}^{\prime}, K^{\prime}\right)$-modules. This means that Theorem 4.3 gives only a partial information about the $(p, q)$-type Hodge component of the modular symbol, $\mathcal{M}^{p, q}(Y)$ for $(p, q) \neq(n, n)$.

Remark 6.4. As Theorem 4.3 suggests, the more representations $\pi \in \widehat{G}$ satisfying (4.3)(ii), the more information we obtain about the modular symbol defined by $\left(G, G^{\prime}\right)$. It might be interesting to investigate in other cases the counterpart in differential geometry (e.g. the middle Hodge component in the case of $\S 5$ where $G / K$ is Hermitian), corresponding to the representation theoretic condition (4.3)(ii), which assures the vanishing of the $\pi$-component in Theorem 4.3.

Remark 6.5. Suppose that $\left(G, G^{\prime}\right)$ is a reductive symmetric pair and defines a modular symbol $\Gamma^{\prime} \backslash G^{\prime} / K^{\prime} \hookrightarrow \Gamma \backslash G / K$, as in (2.6). Y. Tong and S. Wang proved the non-vanishing theorem of the modular symbol (defined on a certain locally constant sheaf) with respect to the $\pi$-component if $\pi \in \widehat{G}$ is a discrete series representation for $G / G^{\prime}([22])$. On the other hand, we have proved the vanishing theorem of the modular symbol with respect to the $\pi$-component, if the restriction $\left.\pi\right|_{G^{\prime}}$ is discretely decomposable (Theorem 4.3). This observation suggests that the assumption on $\pi \in \widehat{G}$ in [22] (i.e. $\pi$ is a discrete series representation for $G / G^{\prime}$ ) and the one in this paper (i.e. $\pi$ is discretely decomposable with respect to $G^{\prime}$ ) should be exclusive. This is true if $G^{\prime}$ is compact because there is no discrete series representation for $G / G^{\prime}$, and is proved in general in [8], Theorem 6.2. For example, if $\left(G, G^{\prime}\right)=\left(S O_{0}(2 n, 2), S O_{0}(2 n, 1)\right)$, then all of discrete series representations for $G / G^{\prime}$ have highest weight vectors (or lowest weight vectors), and therefore, the contribution of the de Rham cohomology group $H_{\text {de }}^{2 n} \operatorname{Rham}(\Gamma \backslash G / K ; \mathbb{C})$ is the $(2 n, 0)$-type Hodge component (or the $(0,2 n)$-type Hodge component), which is the opposite extremal case of the $(n, n)$-type that we have treated in $\S 5$. 


\section{Acknowledgement}

The main part of the manuscript was written while the first author was a guest of the Institut Mittag-Leffler supported by the Royal Swedish Academy of Sciences. He expresses his sincere gratitude to the staff and Professors M. Flensted-Jensen and G. Ólafsson for giving me a warm and stimulating atmosphere of research during the special year 1995-1996 on "Analysis on Lie Groups".

\section{References}

[1] A. Ash and A. Borel, Generalized modular symbols, Cohomology of arithmetic groups and auotmorphic forms, Lecture Notes in Math. 1447, Springer, 1990, pp. 57-76.

[2] A. Borel, Compact Clifford-Klein forms of symmetric spaces, Topology 2 (1963), 111-122.

[3] A. Borel and N. Wallach, Continuous Cohomology, Discrete Subgroups, and Representations of Reductive Groups, Ann. Math. Stud. 94, Princeton U.P., 1980.

[4] G. van der Geer, Hilbert modular surfaces, Ergebnisse der Mathematik und ihrer Grenzgebiete 16, Springer, 1988.

[5] I. M. Gel'fand, M. I. Graev and I. Piateski-Shapiro, Representation Theory and Automorphic Functions, Saunders Math. Books, 1969.

[6] T. Kobayashi, Discrete decomposability of the restriction of $A_{\mathfrak{q}}(\lambda)$ with respect to reductive subgroups and its applications, Invent. Math. 117 (1994), 181-205.

[7] T. Kobayashi, Discrete decomposability of the restriction of $A_{\mathfrak{q}}(\lambda)$ with respect to reductive subgroups II - micro-local analysis and asymptotic $K$-support, Annals of Math. (to appear).

[8] T. Kobayashi, Discrete decomposability of the restriction of $A_{\mathfrak{q}}(\lambda)$ with respect to reductive subgroups III - restriction of Harish-Chandra modules and associated varieties, Invent. Math. 131 (1998), 229-256.

[9] T. Kobayashi, The Restriction of $A_{\mathfrak{q}}(\lambda)$ to reductive subgroups. I, II, Proc. Japan Acad. 69 (1993), 262-267; 71 (1995), 24-26.

[10] T. Kobayashi and K. Ono, Note on Hirzebruch's proportionality principle, J. Fac. Soc. Univ. of Tokyo 37-1 (1990), 71-87.

[11] S. Kudla and J. Millson, Intersection numbers of cycles on locally symmetric spaces and Fourier coefficients of holomorphic modular forms in several complex variables, Inst. Hautes Etudes Sci. Publ. Math. 71 (1990), 121-172.

[12] S. Kumaresan, The canonical $\mathfrak{k}$-types of the irreducible unitary $\mathfrak{g}$-modules with non-zero relative cohomology, Invent. Math. 59 (1980), 1-11.

[13] J.-S. Li, Non-vanishing theorems for the cohomology of certain arithmetic quotients, $J$. reine angew. Math. 428 (1992), 177-217.

[14] Y. Matsushima and S. Murakami, On vector bundle valued harmonic forms and automorphic forms on symmetric spaces, Annals of Math. 78 (1963), 365-416.

[15] N. O. Nygaard, Construction of some classes in the cohomology of Siegel modular threefolds, Compositio Mathematica 97 (1995), 173-186.

[16] T. Oda, On modular forms associated with indefinite quadratic forms of signiture $(2, n-2)$, Math. Ann. 231 (1977), 97-144.

[17] T. Oda, On the poles of Andrianov L-functions, Math. Ann. 256 (1981), 323-340.

[18] T. Oda, Periods of Hilbert modular surfaces, Progress in Math. 19, Birkhäuser, 1982.

[19] T. Oda, A note on a geometric version of the Siegel formula for quadratic forms of signature (2,2k), Science Reports of Niigata University (1984), 13-24.

[20] W. Schmid, Homogeneous complex manifolds and representations of semisimple Lie groups, 
ph. D. Thesis, U.C. Berkeley (1967).

[21] T. Shintani, On construction of holomorphic cusp forms of half integral weight, Nagoya Math. J. 58 (1975), 83-126.

[22] Y. L. Tong and S. P. Wang, Geometric realization of discrete series for semisimple symmetric spaces, Invent. Math. 96-2 (1989), 425-458.

[23] D. Vogan, Jr., Representations of Real Reductive Lie Groups, Progress Math. 15, Birkhäuser, 1981.

[24] D. Vogan, Jr., Unitarizability of certain series of representations, Annals of Math. (1984), 141-187.

[25] D. Vogan, Jr., Unitary Representations of Reductive Lie Groups, Ann. Math. Stud. 118, Princeton U.P., 1987.

[26] D. Vogan and G. Zuckerman, Unitary representations with non-zero cohomology, Comp. Math. 53 (1984), 51-90.

[27] N. R. Wallach, On the unitarizability of derived functor modules, Invent. Math. 78 (1984), $131-141$.

[28] H. Wong, Dolbeault cohomologies and Zuckerman modules associated with finite rank representations, ph. D. dissertation, Harvard University (1991).

T. Kobayashi and T. Oda

Graduate School of Mathematical Sciences

University of Tokyo

Meguro, Komaba, 153

Tokyo, Japan

(Received: December 8, 1996) 